\title{
Recalibrating Software Reliability Models
}

\author{
NNGI.77I \\ Sarah Brocklehurst, P Y Chan, Bev Littlewood \\ IN-6/.CR \\ Centre for Software Reliability \\ City University \\ Northampton Square \\ London EC1V OHB \\ -70364
$36 p$ \\ John Snell \\ Computer Science Department \\ City University \\ Northampton Square \\ London EC1V OHB
}

\begin{abstract}
In spite of much research effort, there is no universally applicable software reliability growth model which can be trusted to give accurate predictions of reliability in all circumstances. Worse, we are not even in a position to be able to decide a priori which of the many models is most suitable in a particular context. Our own recent work has tried to resolve this problem by developing techniques whereby, for eāch program, the accuracy of various models can be analysed. A user is thus enabled to select that model which is giving the most accurate reliability predictions for the particular program under examination. One of these ways of analysing predictive accuracy, which we call the $u$ plot, in fact allows a user to estimate the relationship between the predicted reliability and the true reliability. In this paper we show how this can be used to improve reliability predictions in a completely general way by a process of recalibration. Simulation results show that the technique gives improved reliability predictions in a large proportion of cases. However, a user does not need to trust the efficacy of recalibration, since the new reliability estimates produced by the technique are truly predictive and so their accuracy in a particular application can be judged using the earlier methods. The generality of this approach would therefore suggest that it be applied as a matter of course whenever a software reliability model is used.
\end{abstract}

\footnotetext{
(NASA-CR-156407) RECALIBRATING SOFTWARE

RELIAGILITY MOOELS (City Univ.) $36 \mathrm{p}$

CSCL 098

N90-19763

Unc las

$63 / 61 \quad 0270364$
} 


\section{Introduction}

The earliest attempts to measure and predict the reliability of software occurred about twenty years ago. In spite of considerable research work in the intervening years, there is still no definitive method or model which can be universally recommended as 'best'. Perhaps this should not be surprising. Estimating and predicting software reliability is not easy. Perhaps the major difficulty is that we are concerned primarily with design faults.

This situation is very different from that tackled by the conventional hardware reliability theory. Here the dramatic advances of the past quarter century have come from a concentration on the random processes of physical failure. Thus, for example, we now have a good understanding of how the reliabilities of complex hardware systems depend upon, on the one hand, the detailed system structure, on the other, the reliabilities of the constituent components. The very success of this physical hardware reliability theory, however, is now revealing the importance of design faults to the overall reliability of complex systems. Our ability to use intelligent strategies to minimise the effects of physical failure of components results in a higher proportion of system failures being caused by flawed designs. Such flaws in hardware systems are very similar to software faults: they represent the result of human misunderstandings. It seems likely, as a result of this, that obtaining good methods for measuring the effect of such flaws on hardware system reliability will be as difficult as measuring software reliability.

Software has no significant physical manifestation. Software failures are merely inherent design faults revealing themselves under appropriate operational circumstances. These faults will have been resident in the software since their creation in the original design or in subsequent changes. We currently do not have good theories of how software faults come into being. Presumably such theories would require better understanding of human problem solving and the social processes involved in writing software; if so, we should perhaps look to social and psychological sciences, rather than physics, for solutions. In view of the comparative lack of success of these sciences in arriving at quantitative understanding, it would be wise not to expect any dramatic breakthrough in the short term.

These difficulties notwithstanding, there have been important advances in software reliability modelling recently. In fact, there is now a plethora of models from which the user can choose in order to make reliability estimates and predictions. However, none 
of these has been shown to be applicable in all circumstances, and we are not presently able to decide in a particular context which would be the most appropriate model to use. This presents difficulties for a potential user, who is solely interested in obtaining reliability measures in which he/she can have confidence.

Our own recent work [1] has attempted to tackle this problem by devising means whereby judgements can be made about the accuracy of past predictions on a particular data source. The intention is that a user could apply such techniques, for each data source (program), to the results produced by several models and select the model which has so far performed best by giving the most accurate reliability predictions. It would then be sensible, in the absence of any other information, to use that model for the next prediction on that data source. This 'horses for courses' approach obviates the need for a priori selection of a model, instead each data source is provided with its 'best' model. Indeed, this 'best' model may change as more data is collected.

These new methods of model selection work by analysing the closeness between predicted and actual failure behaviour. In particular, they provide information about two especially important types of departure which we call bias (or ill-calibration) and noise (or variability). The key idea in the present work is that this knowledge of the nature of past errors of prediction can be used to improve future predictions. The techniques to be described here are quite general and are not model-dependent. They will be shown to be effective in improving predictive accuracy in a high proportion of cases, but users need not take this efficacy on trust: their predictive accuracy in a particular case can be analysed, just like any other model, using our earlier techniques [1].

\section{Reliability growth and predictive accuracy}

In its simplest form, the software reliability growth problem concerns the random

variables $T_{1}, T_{2}, \ldots, T_{n}$, representing the execution times between successive failures as a program is being debugged. It is generally assumed that attempts are made at each failure to fix the fault which caused that failure. Models vary in the way that they represent this fault-finding and fixing operation: details of different approaches can be found elsewhere $[1,8,15]$. 
At stage $i$, when observations $t_{1}, t_{2}, \ldots, t_{i-1}$ have been made of the first $i-1$ inter-failure times, the objective is to predict future failure behaviour represented by the unobserved $\mathrm{T}_{\mathrm{i}}, \mathrm{T}_{\mathrm{i}+1}, \ldots$ random variables. Informally, the prediction problem is solved if we can accurately estimate the joint distribution of any finite subset of $T_{i}, T_{i+1} \ldots$. This statement, however, begs the question of what we mean by 'accurately', and it is this issue which forms a major part of our earlier work [1].

In practice, of course, a user will be satisfied with much less than a complete description of all future uncertainty. In many cases, for example, it will be sufficient to know the current reliability of the software under examination. This could be presented in many different forms: the reliability function, $P\left(T_{i}<t\right)$; the current rate of occurence of failures (ROCOF), [3]; the mean (or median) time to next failure (mttf). Alternatively, a user may wish to predict when a target reliability, perhaps to be used as the criterion for termination of testing, will be achieved.

If we accept that prediction is our goal, it can be seen that the usual discussion of competing software reliability growth models is misleading. We should, instead, be comparing the relative merits of prediction systems. A prediction system which will allow us to predict the future $\left(T_{i}, T_{i+1} \ldots\right)$ from the past $\left(t_{1}, t_{2}, \ldots t_{i-1}\right)$ comprises:

(i) the probabilistic model which specifies the distribution of any subset of the $T_{j}$ 's conditional on a (unknown) parameter $\alpha$;

(ii) a statistical inference procedure for $\alpha$ involving use of available data (realisations of $T_{j}$ 's);

(iii) a prediction procedure combining (i) and (ii) to allow us to make probability statements about future $T_{j}$ 's.

Of course, the model is an important part of this triad and it seems unlikely that good predictions can be obtained if the model is not 'close to reality'. However, a good model is not sufficient: stages (ii) and (iii) are vital components of the prediction system. In fact disaster can strike at any of the three stages.

In principle, it ought to be possible to analyse each of the three stages separately so as to gain trust in (or to mistrust) the predictions. Unfortunately, it is our experience that this is not possible. There are several reasons. 
In the first place, the models are usually too complicated for a traditional 'goodness-offit' approach to be attempted. Even the simplest exponential order statistic model [14] does not allow this kind of analysis. This should not surprise us: the goodness-of-fit problem for independent identically distributed random variables is hard in the presence of unknown parameters. The reliability growth context is much worse because of nonstationarity.

Secondly, statistical properties of the estimators of unknown parameters for a nonBayesian analysis of these models are usually not available. For example, several models assume that the software contains only a finite number of faults. There is thus an upper bound on the number of observable $T_{j}$ s. This implies that we cannot even trust the usual asymptotic theory for maximum likelihood (ML) estimators. Their small sample properties are invariably impossibly hard to obtain.

Of course, there is a proper approach to stages (ii) and (iii) in the Bayesian framework. It involves posterior distributions of the parameters at stage (ii) and Bayesian predictive distributions for (iii) (see [2]). Unfortunately, this does present some analytical difficulties for the popular software reliability growth models. However, with recent advances in Bayesian numerical techniques [18], coupled with powerful personal computers, this picture may change in the near future.

Finally, it could be argued that there are models which are 'obviously' better than others because of the greater plausibility of their underlying assumptions. We find this a dubious proposition. Certainly, the assumptions of some models seem overly naive and it might be reasonable to discount them. However, this still leaves others which cannot be rejected a priori. It is our belief that understanding of the processes of software engineering is so imperfect that we cannot even choose an appropriate model when we have an intimate knowledge of the software under study. At some future time it may be possible to match a reliability model to a program via the characteristics of that program, or even of the software development methodology used. This is not currently the case.

Where does this leave a user, who merely wants to obtain trustworthy reliability metrics for his current software project? Our view is that there is no alternative to a direct examination and comparison of the quality of the predictions emanating from different complete prediction systems. In [1] we have described several ways in which this can be done, the most important tools being the $u$-plot and the prequential likelihood. The key idea in each case is that a comparison is made between what has been predicted and 
what is (later) actually observed. We believe that this emulates how a user would informally gain confidence in a sequence of predictions.

For simplicity we shall concentrate on prediction of the next time to failure $T_{i}$, based on observations $t_{1}, t_{2}, \ldots, t_{i-1}$. The $u$-plot uses the predictor $\hat{F}_{i}(t)$, the estimate of the distribution function $F_{i}(t)=P\left(T_{i} \leq t\right)$, via ${ }^{\top}$

$$
\mathrm{u}_{\mathrm{i}}=\hat{\mathrm{F}}_{\mathrm{i}}\left(\mathrm{t}_{\mathrm{i}}\right)
$$

where $t_{i}$ is the later-observed realisation of the random variable $T_{i}$. Thus $u_{i}$ is the probability integral transform of the observation using the predictive distribution function. If the sequence of predictions $\left\{\hat{F}_{i}\left(t_{i}\right)\right\}$ is good, it is easy to see that the sequence $\left\{u_{i}\right\}$ should look like a random sample from a $U(0,1)$ distribution [1]. There are various types of departure from such an appearance which might show themselves; here we shall only be concerned with whether the $\left\{u_{i}\right\}$ sequence looks uniformly distributed. We shall do this via the $u$-plot which is the sample cumulative distribution (cdf) function of the $u_{i}$ sequence. The departure of this plot from the cdf of $U(0,1)$, the line of unit slope, is an indication of a departure of the prediction system from accuracy. We can use the Kolmogorov distance, that is the maximum vertical deviation, as a measure of this departure and use standard tables to determine whether or not it is statistically significant.

Figure 1 shows u-plots for Jelinski-Moranda [10] and Littlewood-Verrall [13] models making predictions on a data set, called S1 [17], analysed in [1]. These plots are each based on 86 predictions: $\hat{\mathrm{F}}_{51}(\mathrm{t})$ through $\hat{\mathrm{F}}_{136}(\mathrm{t})$. The Kolmogorov distances are 0.205 (JM) and $0.150(\mathrm{LV})$. The first is significant at the $1 \%$ level, suggesting very poor prediction from JM; the second is significant at $5 \%$, which suggests that this model is also performing poorly but is somewhat superior to JM.

More importantly for our present purposes, the shape of the plots tells us that JM is making predictions which are too optimistic, whilst LV predictions are too pessimistic. This can be seen as follows. The JM plot is everywhere above the line of unit slope (the true $U(0,1)$ cdf), so there are too many small $u_{i}$ values. But consistently too small $u$ values tells us that the model is underestimating the chance of small times between failure, i.e. the model is too optimistic. A similar argument shows that a plot which is almost everywhere below the line of unit slope, such as $\mathrm{LV}$, is too pessimistic. 
If we knew that these deviations between predicted and actual behaviour were consistent, we could attempt to measure the degree of optimism (or pessimism) and improve future predictions by taking account of this tendency. It is this idea which we shall develop in the next section. Before we do that, we shall briefly describe the prequential likelihood function (PL) which is a general mechanism for comparing the accuracy of prediction systems.

The PL is defined as follows. The predictive distribution $\hat{F}_{i}(t)$ for $T_{i}$ based on $t_{1}$, $t_{2}, \ldots, t_{i-1}$ will be assumed to have a probability density function ( $p d f$ )

$$
\hat{f}_{i}(t)=\hat{F}_{i}^{\prime}(t)
$$

For predictions of $T_{j+1}, T_{j+2}, \ldots, T_{j+n}$, the prequential likelihood is

$$
P L_{n}=\prod_{i=j+1}^{j+n} \hat{f}_{i}\left(t_{i}\right)
$$

A comparison of two prediction systems, $A$ and $B$, over a range of predictions of $T_{j+1}$, $\mathrm{T}_{\mathrm{j}+2}, \ldots \mathrm{T}_{\mathrm{j}+\mathrm{n}}$, can be made via their prequential likelihood ratio

$$
\begin{aligned}
& \operatorname{PLR}_{n}=\frac{\prod_{i=j+1}^{j+n} \hat{f}_{i} A_{\left(t_{i}\right)}}{j+n} \\
& \prod_{i=j+1} \hat{f}_{i} B\left(t_{i}\right)
\end{aligned}
$$

Notice how, in a fashion analogous to the calculation of the u sequence, the individual contributions to the prequential likelihood are obtained by substitution into the predictor pdf for $T_{i}$ of the the later-observed realisation $t_{i}$. Dawid [7] shows that if $\operatorname{PLR}_{n} \rightarrow \infty$ as $n \rightarrow \infty$, prediction system $B$ is discredited in favour of $A$. For the finite samples with which we inevitably have to deal, we shall argue that $P_{L} R_{n}$ increasing consistently suggests the superiority of A over B. In [1] we give intuitive reasons why the PL works. Specifically we show that consistent bias or noisiness of a prediction system will tend to give a smaller PL than would otherwise be the case.

To summarise, the PLR can be regarded as a general procedure for choosing the best prediction system for a particular data source. The u-plot is a means of indicating a 
particular kind of consistent inaccuracy of prediction which could be a contributory factor in poor predictive accuracy. Thus a poor u-plot might suggest that poor predictive accuracy (represented by a poor prequential likelihood) is due to consistent bias. For such a case, we shall show in the next section how it is possible to remove the bias and so improve the accuracy of reliability predictions.

\section{Recalibration of predictions}

Consider a prediction $\hat{\mathrm{F}}_{\mathrm{i}}(\mathrm{t})$ of the random variable $\mathrm{T}_{\mathrm{i}}$, when the true (unknown) distribution is $F_{i}(t)$. Let the relationship between these be represented by the function $\mathrm{G}_{\mathrm{i}}$ where

$$
F_{i}(t)=G_{i}\left[F_{i}\left(t_{i}\right)\right]
$$

Obviously, if we knew $G_{i}$ we could recover the true distribution of $T_{i}$ from the inaccurate predictor, $\hat{\mathrm{F}}_{\mathrm{i}}\left(\mathrm{t}_{\mathrm{i}}\right)$. The key notion in our recalibration approach is that in many cases the sequence $\left\{G_{i}\right\}$ is approximately stationary, i.e. it is only slowly changing in $i$.

If the sequence were completely stationary, i.e. $G_{i}=G$ for all $i$, we would have a more precise interpretation of the idea of 'consistent bias' used in the previous section. We would also have the possibility of estimating the common $\mathrm{G}$ from past predictions and using it to improve the accuracy of future predictions.

Of course, in practice such complete stationarity is unlikely to be achieved. However, it does seem to be the case that the sequence changes only slowly in many cases. This opens up the possibility of approximating $\mathrm{G}_{\mathrm{i}}$ with an estimate $\mathrm{G}_{\mathrm{i}}{ }^{*}$ and so forming a new prediction

$$
\hat{F}_{i}^{*}\left(t_{i}\right)=G_{i}^{*}\left[\hat{F}_{i}\left(t_{i}\right)\right] .
$$

A suitable estimator for $G_{i}$ is suggested by the observation that $G_{i}$ is the distribution function of $\mathrm{U}_{\mathrm{i}}=\hat{\mathrm{F}}_{\mathrm{i}}\left(\mathrm{T}_{\mathrm{i}}\right)$. We shall therefore base our estimate $\mathrm{G}_{\mathrm{i}}{ }^{*}$ on the u-plot, calculated from predictions which have been made prior to $T_{i}$, which is the sample cdf formed from the $u_{j} s$ for $j<i$. The new prediction (5) recalibrates the raw model output, 
$\hat{F}_{i}\left(t_{i}\right)$, in the light of our knowledge of the accuracy of past predictions for the data source under study. The new procedure is therefore a truly predictive one, 'learning' from past errors.

The simplest form for $\mathrm{G}_{\mathrm{i}}{ }^{*}$ is the $\mathrm{u}$-plot with steps joined up to form a polygon (Figure 2). Later we shall consider a version which is smoothed using a spline technique. The complete procedure for forming a recalibrated prediction for the next time to failure, $\mathrm{T}_{\mathrm{i}}$, is then:

Stage 1 Check that error in previous predictions is approximately stationary. (See [1] for a plotting technique, the y-plot, which detects nonstationarity, although we shall see later that recalibration often works well even in the presence of non-stationarity)

Stage 2 Find $u$-plot for predictions made before $T_{i}$, i.e. based on $t_{1}, t_{2}, \ldots t_{i-1}$, and join up the steps to form a polygon, $\mathrm{G}_{\mathrm{i}}^{*}$.

Stage 3 Use the basic prediction system to make a 'raw' prediction, $\hat{\mathrm{F}}_{\mathrm{i}}\left(\mathrm{t}_{\mathrm{i}}\right)$.

Stage $4 \quad$ Recalibrate the raw prediction using (5).

This whole procedure can be repeated at each stage so that the functions $\mathrm{G}_{\mathrm{i}}{ }^{*}$ used for recalibration will be based on more information about past errors as $\mathrm{i}$ increases. For the simple joined-up u-plot this is not computationally onerous: by far the greatest computational effort is needed for the statistical inference procedures used to obtain the raw model predictions.

It is important to emphasise that the procedure described above does in fact produce a genuine prediction system in the sense described earlier: at each stage we are using only past observations to make predictions about the unobserved future failure behaviour.

Figure 3 shows the effect of recalibration on the predictions made in Figure 1. In the case of the JM model it is known that the raw predictions are too optimistic, and the recalibration makes them less optimistic; in the case of LV, which is initially too pessimistic, the recalibrated version is now less pessimistic. These conclusions are confirmed in the more formal analysis based on the u-plot technique: for $\mathrm{JM}^{*}$ the Kolmogorov distance of the $\mathrm{u}^{*}$-plot is 0.119 (compared with 0.205 for the raw predictions), for $\mathrm{LV}^{*}$ it is 0.089 (compared with 0.150 ). Not only are these an 
improvement in each case, the distances are now no longer statistically significant at the $10 \%$ level.

Notice that, although Figure 3, for simplicity, only shows median predictions, the recalibration is working on the complete predictive distribution. Thus it could be expected to improve other reliability estimates, such as the rate of occurence of failures, in the examples shown here. The recalibration procedure changes the complete shape of the distribution and can therefore correct for far more subtle errors than the mainly simple 'optimism' or 'pessimism' of these examples.

Figure 4 shows an analysis of a data set, SS3 from [17], which exhibits startling disagreement between raw predictions from $\mathrm{JM}$ and LV models. In fact, in an analysis of this data using nine models [5], it can be seen that seven of them are in close agreement with one another and are close to the $\mathrm{JM}$ plot in Figure 4; the remaining two are close to the LV plot in Figure 4. A user might conclude that the seven models which give similar answers are closer to the truth than the more isolated pair, but this would be wrong. In fact for this data set none is giving acceptable answers. This is shown by the u-plots for JM and LV predictions in Figure 5. Clearly, the JM predictions are optimistic, and those from LV pessimistic. The effect is a gross one, as can be seen from the Kolmogorov distances, 0.272 (JM) and $0.238(\mathrm{LV})$, which are very highly significant (well beyond the $1 \%$ level, the highest tabulated). The prequential likelihood shows that LV is superior to JM [1], but neither of them, nor any other model we have used, gives accurate reliability predictions for this data source.

The detailed shape of the u-plots in Figure 5 is interesting. As was stated above, the most notable feature is the extreme optimism or pessimism. However, this is not a simple effect in either case. For JM the behaviour of the plot at each extremity suggests too many very small u values and too many very large ones. For LV there seem to be too many fairly large u's and too few u's near to 1.0. Thus, although the statements above about optimism and pessemism are correct to a first approximation, a more detailed analysis shows that the u-plots are giving precise information about the incorrect shapes of the complete predictive distributions. It can therefore be seen how the recalibration procedure based on such u-plots can effect subtle changes in the complete estimated distribution function for the random variable $T_{i}$.

The recalibration technique works dramatically well for this data. Table 1 shows a comparison between raw model predictions and recalibrated predictions for the following nine models: JM (Jelinski-Moranda, [10]), BJM (Bayesian Jelinski- 
Moranda, [11]), GO (Goel-Okumoto, [9]), MO (Musa-Okumoto, [16]), D (Duane, [6]), L (Littlewood, [12]), LNHPP (Littlewood non-homogeneous Poisson process, [1]), LV (Littlewood-Verrall, [13]), and KL (Keiller-Littlewood, [1]).

All nine raw u-plots have Kolmogorov distances which are significant well beyond the tabulated $1 \%$. After recalibration, all the distances have been more than halved and none are significant at this high level. Figure 6 shows the dramatic improvement given by recalibration on the JM and LV u-plots in comparison with the raw predictions (see Figure 5). The differences in the detailed median predictions (only for JM and LV again, for simplicity) can be seen by comparing Figures 4 and 7 . There is much closer agreement between the recalibrated models than between the raw ones.

In both the above examples there is evidence that prediction systems which were in disagreement have been brought into closer agreement by the recalibration technique. Much more important, however, we have objective evidence from the comparison of $\mathrm{u}$ plot with $u^{*}$-plot that recalibrated predictions are less 'biased' than the raw ones.

These results are encouraging for the efficacy of the recalibration approach, but they are not sufficient grounds for assuming, even in the two examples here, that the recalibrated predictions should be preferred to the raw ones. It may be that the advantage of less bias has been bought at the expense of some other deviation between predicted and actual reliability. We have suggested in the previous section that the prequential likelihood should be used as arbiter between competing prediction systems for any particular data source. It would seem appropriate, therefore, to judge whether a raw or recalibrated prediction system is objectively best by comparing their prequential likelihoods for a series of predictions. Unfortunately this presents problems for recalibrated predictions which are based on the simple polygonal joined-up u-plots suggested above. The reason is somewhat 'technical' and is due to the fact that the PL uses the probability density function of the predictive distribution:

$$
\begin{aligned}
\mathrm{PL}_{n}^{*} & =\prod_{i=j+1}^{j+n} \hat{f}_{i}^{*}\left(t_{i}\right)=\prod_{i=j+1}^{j+n} g_{i}^{*}\left(\hat{F_{i}}\left(t_{i}\right)\right) \cdot \hat{f}_{i}\left(t_{i}\right) \\
& =\prod_{i=j+1}^{j+n} g_{i}^{*}\left(u_{i}\right) \cdot \hat{f}_{i}\left(t_{i}\right)
\end{aligned}
$$

from (5), letting $\mathrm{gi}^{*}$ denote the derivative of $\mathrm{G}_{\mathrm{i}}{ }^{*}$. 
Unfortunately, since $\mathrm{G}_{\mathrm{i}}{ }^{*}$ is a polygon, its derivative $\mathrm{gi}^{*}$ is discontinuous. This means that $\hat{\mathrm{f}}_{\mathrm{i}}{ }^{*}$ is also discontinuous: Figure 8 shows an example of this problem. This discontinuity generally causes PL to report badly on the predictive accuracy of a recalibrated model in competition with the raw version. A user might therefore conclude that recalibration had made the predictions less accurate. We think this can be misleading. It is true that it would be unreasonable to believe that the true predictive pdf is grossly discontinuous; the rejection of such a pdf by the PL criterion is therefore strictly correct. However, in practice users are not directly interested in predictive pdfs but in probabilities. Such probabilities will be obtained from the pdf by integration, which has the effect of smoothing out the discontinuity. It is therefore perfectly possible for PL to reject a recalibrated prediction system in favour of the raw version, even when the recalibrated (probability) predictions are the most accurate. A rejection in such circumstances is, we believe, unfair: a user needs to know which prediction system is performing best for the kinds of prediction he is likely to make.

There are two ways forward which will be described in the next two sections.

The first approach attempts to decide whether recalibration can be trusted to give improved results in a wide class of circumstances by comparing both recalibrated and raw predictions with the true reliability when this is known. In practice, of course, such knowledge of the truth is not available so we shall have to use simulated interfailure times. We shall show that in a high proportion of cases the recalibrated prediction system is superior to the raw one. However, as might be expected, this is not always the case.

Our second approach, therefore, applies a smoothing to the polygonal $\mathrm{G}_{\mathrm{i}}{ }^{*}$ in order to give a continuous recalibrated predictive pdf. This allows the use of PL as a criterion for judging which prediction system is giving most accurate results. Use of this smoothing is computationally more intensive than use of the simple joined-up u-plot.

A user therefore has a choice: appeal to the general efficacy of the approach as demonstrated by the simulation results based on the simple recalibration technique, or use the smoothed version and use PL to decide whether recalibration is working in the particular example under study. 


\section{Simulation results}

The simulation experiment [4] consisted of generating 100 realisations of the interfailure time sequence $t_{1}, t_{2}, \ldots t_{100}$ from each of the models $J M, L, L V, K L$ and $D$, with constant parameters being used for each model. These data sets were then analysed using the 'wrong' models: thus, for example the JM data set was analysed using the $\mathrm{L}, \mathrm{LV}, \mathrm{KL}$ and $\mathrm{D}$ models.

The model parameters were estimated based on $t_{1}, t_{2}, \ldots t_{j-1}$, to obtain $\hat{F}_{j}(t)$ for $j=$ $20, \ldots$ 101. Then, for $i=40, \ldots 101$, the u-plot using $u_{j}=\hat{F}\left(t_{j}\right)$, for $j=20, \ldots i-1$, was used to obtain $G_{i}^{*}$ and hence $\hat{F}_{i}^{*}(t)$. It was thus possible to compare the known true $F_{i}(t)$ with the raw predictor $\hat{F}_{i}(t)$ and with the recalibrated predictor $\hat{F}_{i}^{*}(t)$ for $i=$ $40, \ldots, 101$.

In a particular case a user is interested in knowing whether the raw or recalibrated predicted distribution is closer to the true one. There are various ways we could examine the differences between predicted and true distributions. Perhaps the most obvious is a direct measure of the distance between the two functions, such as the Kolmogorov distance. This is defined as follows. For raw predictions let $\hat{d}_{\mathrm{i}}(\mathrm{t})=\mathrm{F}_{\mathrm{j}}(\mathrm{t})$ - $F_{i}(t)$ and for recalibrated $\hat{d}_{i}{ }^{*}(t)=\hat{F}_{i}{ }^{*}(t)-F_{i}(t)$, both for $i=40, \ldots$ 101. The Kolmogorov distances are $\hat{\mathrm{k}}_{\mathrm{i}}=\sup _{\triangleright 0}\left|\hat{\mathrm{d}}_{\mathrm{i}}(\mathrm{t})\right|=\left|\hat{\mathrm{d}}_{\mathrm{i}}(\tau)\right|$ and $\hat{\mathrm{k}}_{\mathrm{i}}^{*}=\sup _{\triangleright 0}\left|\hat{\mathrm{d}}_{\mathrm{i}}{ }^{*}(\mathrm{t})\right|=$ $\left|\hat{\mathrm{d}}_{\mathrm{i}}^{*}(\gamma)\right|$. A simpler procedure is to merely check whether the recalibrated or raw median is closer to the true one.

The first analysis concerns only predictions of $\mathrm{T}_{101}$; there are 2000 such predictions in the experiment. If we consider those predictions of $\mathrm{T}_{101}$ for which the $\mathrm{u}$-plot (based on predictions prior to $\mathrm{T}_{101}$ ) was significant at the $5 \%$ level, indicating that there was evidence of bias, $89 \%$ of the recalibrated predictions were superior to the corresponding raw ones. This figure rises to $92 \%$ if we only recalibrate for u-plots which are significant at the $1 \%$ level.

Even when we recalibrated regardless of the u-plot evidence, the recalibrated predictions improved on raw ones in $61 \%$ of cases. Here there will be many cases where raw predictions are close to the truth; then we would not expect the recalibration to introduce an improvement and the recalibrated and raw predictions should be close to one another. However, since the recalibrated predictive distribution is polygonal ('lumpy'), the Kolmogorov distance (which compares the maximum deviations of the 
two predictions from the truth) will tend to discriminate against the recalibration in favour of the raw prediction. This figure of $61 \%$ can therefore be thought of as a conservative one.

Other simple comparisons between recalibrated and raw predictions are fairer in this situation. For example, the recalibrated median is closer than the raw one to the true median in $70 \%$ of these cases. This figure rises to $91 \%$ when we recalibrate only for $u$ plots significant at $5 \%$, and $94 \%$ when we recalibrate only for u-plots significant at $1 \%$.

These results for $\mathrm{T}_{101}$ are supported by the more extensive recalibrations of the predictions of $\mathrm{T}_{40}, \ldots, \mathrm{T}_{101}$ : here recalibrated medians are closer to the true one in $86 \%$ of cases when the u-plot at stage 100 was $5 \%$ significant, and are closer in $93 \%$ of cases when the u-plot is significant at $1 \%$.

In summary, even when we blindly used the recalibration on all predictions, there was an improvement in about 7 out of 10 cases. More importantly, when we adopted the more rational and discriminating approach of only using the technique when the u-plot analysis suggested recalibration might be fruitful (by indicating the presence of 'bias'), there was improvement about 9 out of 10 times.

Of course, we do not know whether our simulated data was typical of real software reliability data. Indeed, since we were generating data according to several models with very different underlying assumptions, some of the data sets are likely to be unrealistic. However, we believe that these results are encouraging for the general power of the approach.

In practice a user might wish to have more than a belief in the general efficacy of the approach: he needs to know that it is working for the particular data source under examination. The obvious approach is to use the methods of analysis of predictive quality [1] discussed earlier. In the next section we show how this can be done.

\section{$5 \quad$ Parametric spline smoothing}

The u-plot is merely the sample cdf of the observed u's. Thus the problem of estimating the approximately stationary function $G_{i}$ in (4) is simply the problem of 
obtaining an estimate of a cdf from a finite random sample. There are several ways in which this can be done so that the estimator is differentiable and so has a smooth pdf. We could, for example, fit an appropriate parametric family of distributions to the data. An example is the family of $\operatorname{Beta}(\alpha, \beta)$ distributions with pdf

$$
f(u)=u^{\alpha-1}(1-u)^{\beta-1} / B(\alpha, \beta) \quad 0 \leq u \leq 1
$$

This is a fairly flexible family, but it is not sufficiently wide to represent all the general shapes of u-plots which we have encountered in practice (see [5] for an example). This seems likely to be a problem with other candidate parametric families of distributions. Another, less important, difficulty is that the evaluation of the cdf is not easy for certain regions of the parameter space.

The need for a method of fitting a very general class of u-plot data suggests the use of parametric splines, which are widely used in computer graphics because of their versatility. We shall use the cumulative chord as the parameter, whereupon the spline is defined as follows. Let $\left\{x_{i}, y_{i}\right\}$, for $i=1,2, \ldots, r$, denote the $r$ points of the $u$-plot to which we want to fit the spline, and let

$$
p_{i}{ }^{\prime}=p_{i-1}{ }^{\prime}+\left[\left(x_{i}-x_{i-1}\right)^{2}+\left(y_{i}-y_{i-1}\right)^{2}\right]^{1 / 2}
$$

with $\mathrm{P}_{0}^{\prime}=0, \mathrm{x}_{0}=0$ and $\mathrm{y}_{0}=0$; i.e. $\mathrm{p}_{\mathrm{i}}{ }^{\prime}$ is the distance from the origin, along the polygon, to the $i$ th point. Here $x_{i}$ is the ith order statistic of the $u$ 's and $y_{i}$ is the height of the $u$-plot at $x_{i}$. For convenience we shall use the normalised chord

$$
\mathrm{p}_{\mathrm{i}}=\mathrm{p}_{\mathrm{i}}{ }^{\prime} / \mathrm{p}_{\mathrm{r}}{ }^{\prime}
$$

so that both parametric functions will have domain $[0,1]$.

We now have two sets of data, $\left\{x_{i}, p_{i}\right\}$ and $\left\{y_{i}, p_{i}\right\}$, to each of which we fit a three knot least-squares cubic spline; call these $x=x(p)$ and $y=y(p)$. These splines are each constrained so that $x(p)$ and $y(p)$ are strictly increasing functions taking values between 0 and 1 for $p$ in $(0,1)$, with $x(0)=y(0)=0$ and $x(1)=y(1)=1$. It follows that the function defined parametrically as $(x(p), y(p))$ is also strictly increasing between 0 and 1. We call this function the parametric spline and it has the properties of a cdf. More importantly for our needs, it is everywhere differentiable with a smooth derivative. This means that if we use this function to recalibrate software reliability predictions we are certain to obtain a smooth recalibrated predictive density. We can therefore use 
prequential likelihood as a criterion of predictive accuracy and be confident that we shall not encounter the difficulties we met with the polygonal joined-up u-plot.

Clearly, using this spline is more tedious than recalibrating predictions from the joinedup u-plot; details can be found in [5]. However, run times are generally much less than are required for the original raw predictions. Since these raw predictions must always be computed, the small overhead involved in using the spline is worthwhile. Most importantly this technique allows a user to determine, via prequential analysis, whether the recalibrated predictions are objectively better than the raw ones for a particular data source. It also similarly allows comparisons to be made between different recalibrated prediction systems. Such knowledge about the performance in a particular instance is more valuable than the general assertions of efficacy which come from the earlier simulation exercise.

To distinguish it from the earlier polygonal $G^{*}$, we shall denote the spline smoothed recalibrating function by $G^{* *}$. The recalibrated predictions are then

$$
\hat{\mathrm{F}}_{\mathrm{i}}^{* *}(\mathrm{t})=\mathrm{G}_{\mathrm{i}}^{* *}\left[\hat{\mathrm{F}_{\mathrm{i}}}(\mathrm{t})\right]
$$

Table 2 shows the u-plot and y-plot Kolmogorov distances for the same data sets as those used in Table 1. It can be seen that the entries in the two tables are very similar. This is to be expected since the spline recalibrated predictive distribution function is designed to be a smooth function close to the joined-up recalibrated predictive distribution. If these two functions are close, the u's based on them will be close and thus so will the plots. In practical terms this means that the predictions of probabilities from the two techniques will be very similar, and in particular their medians are very close (compare Figure 9 with Figure 7). However, their predictions of probability densities will be very different: it is this difference we wish to exploit in the use of the prequential likelihood for the spline version.

In Figure 10 the evolution of the prequential likelihood ratios is shown for the various recalibrated predictions against raw model predictions. Notice how, for LV, the prequential likelihood seems to be suggesting that the joined-up recalibrated predictions are worse than the raw ones. This is a dramatic example of the effect of the discontinuity of joined-up recalibrated probability densities upon the likelihood: it causes a spurious rejection of these recalibrated predictions in favour of those from the raw model. That this is, indeed, spurious can be seen from the behaviour of the spline recalibrated predictions: there is overwhelming evidence that the $\mathrm{LV}^{* *}: \mathrm{LV}$ prequential 
likelihood ratio is increasing rapidly (it has reached more than $e^{40}$ during these predictions!). A user could therefore be very confident that the $\mathrm{LV}^{* *}$ predictions here are more accurate than the LV ones.

A comparison of $\mathrm{JM}^{* *}$ and $\mathrm{JM}$ is even more dramatic: the PLR reaches $\mathrm{e}^{90}$ over the range of predictions shown. This is partly due to the fact that raw JM predictions are significantly less accurate than those of raw LV (although both are bad from u-plot evidence). Thus JM starts off with more room for improvement. In fact, after recalibration, the two spline predictors $\mathrm{LV}^{* *}$ and $\mathrm{JM}^{* *}$ have comparable accuracy on the prequential likelihood evidence, with slight evidence of superiority for $\mathrm{JM}^{* *}$.

Figure 11 shows an example of recalibrated probability density functions at stage 278 in the SS3 data set.. The two raw predictive densities from LV and JM disagree greatly, but after recalibration there is close agreement between $\mathrm{LV}^{* *}$ and $\mathrm{JM}^{* *}$. This is illustrated even more dramatically in Figure 12 which shows predictive densities for stage 121 in the S1 data. Notice here the curious mode which appears in each predictive density after recalibration. Neither of the raw predictive densities (exponential for JM, Pareto for LV) can have a non-zero mode, which suggests that the 'learning' from past errors can give an insight not present in the raw models. What is particularly striking, we believe, in figures like this is not only the close agreement of the two predictions after recalibration, but how dramatically these differ from the raw predictions.

These figures give some indication of the power of the method to change fundamentally the raw prediction, on the evidence of analysis of past predictive error. Thus the improvements in simple summary statistics shown in the median plots (Figures 3,7,9) are merely the tip of an iceberg: when recalibration works it will do so in very general ways and a user could reasonably expect all reliability measures to improve in accuracy.

\section{Retrodictive recalibration}

The recalibration technique described in this paper is based on an analysis of the accuracy of similar predictions at earlier stages in the acquisition of data from testing a program. Thus when we came to recalibrate the prediction of $T_{101}$ it was necessary to make predictions of $T_{20}, T_{21}, \ldots, T_{101}$ (each based only on the data observed prior to 
making the prediction) in order to calculate the $\mathrm{G}_{\mathrm{i}}^{*}$ (or $\mathrm{G}_{\mathrm{i}}{ }^{* *}$ ), $\mathrm{i}=40, \ldots, 101$, which transforms the raw prediction, $\hat{F}_{i}(t)$. For all the models each such prediction is quite computationally intensive, so a single recalibration can require considerable effort. If recalibration is to take place at each stage as each new inter-failure time is observed, then of course this overhead disappears, since it will be necessary to calculate each raw prediction anyway.

However, the problem seemed sufficiently important that we examined a retrodictive recalibration procedure which only needs a single basic calculation (e.g. maximisation of a likelihood function) for each recalibration. For those models using maximum likelihood estimation of the parameters this scheme works as follows. To predict $T_{101}$, we use all available data, $t_{1}, \ldots, t_{100}$, to calculate an estimate of the model parameters. This is used, of course, to obtain the raw prediction of $\mathrm{T}_{101}$. It is also used to retrodict (i.e. "predict" the past) $T_{1}, T_{2}, \ldots T_{100}$. Since we have the actual observations of this past, we can compare the retrodictions with these in the same way that we do with genuine predictions. In particular we can form the retrodictive $u$-plot and use this to recalibrate the raw prediction of $\mathrm{T}_{101}$.

Unfortunately, this procedure seems to be useless! The reason is fairly subtle. It seems to be the case that a prediction of $T_{i}$, based on $t_{1}, \ldots, t_{i-1}$, can be error in different ways from a retrodiction of $T_{j}(j<i)$ also based on $t_{1}, \ldots, t_{i-1}$. More precisely, the approximate stationarity in the errors of prediction of $T_{i}$ (based on $t_{1}, \ldots, t_{i-1}$ ) as we vary $i$ is very different from the approximate stationarity of errors of prediction of $T_{j}$ (based on $t_{1}, \ldots, t_{i}$ ) as we vary $j$ for fixed $i$. It seems that we can expect to obtain the first kind of approximate stationarity, but not the second: it is, of course, such approximate stationarity which underpins the basic idea of recalibration.

Once again this seems to suggest that in assessing software reliability we must be careful of making unfounded generalisations. Just as we cannot assume that a model performing accurately on one data set necessarily will give good performance on another [1], so we cannot assume that information gained from an analysis of the accuracy of one type of prediction will necessarily be trustworthy for another. Although these remarks are based on the evidence of retrodictive error being a poor guide to one-step-ahead prediction, it is likely that the implications are more far reaching. For example, the predictive recalibration method for one-step-ahead predictions may not be effective for predictions further ahead. Thus if we wished to recalibrate a raw 20-step-ahead prediction it may be necessary use a form of the $G$ 
function which is itself based on a comparison of 20-step-ahead raw predictions with actual (later observed) data. We hope to investigate issues of this kind in future work.

\section{Discussion and conclusion}

We have shown that recalibration can be a powerful technique for improving the accuracy of software reliability growth predictions. The technique is completely general, and in particular is not model-dependent: it can be applied to any predictive scheme. It can also be used for different types of prediction, but it should be remembered that recalibration should be based on past predictions of the same type.

Our simulation results for the simple joined-up $G^{*}$ suggest that it offers an improvement in accuracy over the original models in a high proportion of cases. This alone would be sufficent reason for advocating that it be applied as a matter of course to all models: essentially doubling the number of prediction systems available to the user.

As we have demonstrated elsewhere [1], a user cannot select a model a priori from this plethora of available models and know that it is the best for the job. Instead, it is necessary to apply all available models to each data source and use the techniques described in [1], principally the prequential likelihood, to select the one which is giving most accurate reliability predictions for the particular data source (program) under study.

To make this method of discriminating between reliability prediction systems work for recalibrated models, we have introduced the notion of a spline-smoothed recalibrated prediction. The user is now in a position to apply several models, and their recalibrated versions, to his/her data and select that which is objectively performing best. We believe that this eclectic approach should in future be standard practice.

Our results give a new insight into reliability growth modelling. It can now be seen as essentially a two stage process: first capturing the long term trend and then using these new ideas to estimate local behaviour. A rich class of new models could be formed from a distribution-free fitting of trend, followed by a later analysis of detailed probabilistic structure along the lines described above. We are currently investigating these possibilities: early results are encouraging. 


\section{Acknowledgements}

This work was partially supported by the SERC and Alvey Directorate (SB and BL) under project SE-072, partially by ICL plc (PYC) and partially by National Aeronautics and Space Administration under grants number NAG-172 and NAG 1-771. 


\section{References}

[1] Abdel-Ghaly, A. A., Chan, P. Y., Littlewood, B., "Evaluation of competing software reliability predictions", IEEE Trans Software Engineering, SE-12, pp950-967, 1986.

[2] Aitchison, J., Dunsmore, I. R., Statistical Prediction Analysis, Cambridge University Press, Cambridge, 1975.

[3] Ascher, H., Feingold, H., Repairable Systems Reliability, Lecture Notes in Statistics, No 7, Marcel Dekker, New York, 1984.

[4] Brocklehurst, S., "On the effectiveness of adaptive software reliability modelling", CSR Technical Report , Centre for Software Reliability, City University, London, October 1987.

[5] Chan, P. Y., Software Reliability Prediction, $\mathrm{PhD}$ thesis, City University, London, 1986.

[6] Crow, L. H., "Confidence interval procedures for reliability growth analysis", Tech Report No 197, US Army Material Systems Analysis Activity, Aberdeen, Md, 1977.

[7] Dawid, A. P., "The well-calibrated Bayesian", (with discussion) J Amer Statist Assoc, 77, pp 605-613, 1982.

[8] Goel, A. L., Bastani, F. B., IEEE Trans Software Engineering Special Issue on Software Reliability, SE-11, no. 12, 1985 and SE-12, no. 1, 1986

[9] Goel, A. L., Okumoto, K. 'Time-dependent error-detection rate model for software reliability and other performance measures', IEEE Trans Reliability, Vol R-28, pp 206-211, 1979.

[10] Jelinski, Z., Moranda, P. B., "Software reliability research", in Statistical Computer Performance Evaluation (W Freiberger, ed.), New York, Academic Press, 1972, pp. 465-484. 
[11] Littlewood, B., Sofer, A., "A Bayesian modification to the Jelinski-Moranda software reliability model", Software Engineering Journal, 2, pp30-41, 1987.

[12] Littlewood, B., "Stochastic reliability growth: a model for fault-removal in computer programs and hardware designs", IEEE Trans. Reliability, Vol R-30, 4, Oct 1981, pp 313-320.

[13] Littlewood, B., Verrall, J. L., "A Bayesian reliability growth model for computer software", J Royal Statist. Soc., C (Applied Statistics), 22, 1973, pp. 332-346.

[14] Miller, D. R., "Exponential order statistic models of software reliability growth", Tech Report, T-496/84, George Washington University, Washington DC, 1984.

[15] Musa, J. D., Iannino, A., Okumoto, K., Software Reliability: Measurement, Prediction and Application, McGraw-Hill, New York, 1987.

[16] Musa, J. D., Okumoto, K., "A logarithmic Poisson execution time model for software reliability measurement", Proc 7 th International Conference on Software Engineering, IEEE Computer Society, New York, 1984, pp230-238.

[17] Musa, J. D., "Software reliability data", report available from Data Analysis Centre for Software, Rome Air Development Center, Rome NY, 1979.

[18] Smith, A. F. M., Skene, A. M., Shaw, J. E. H., Naylor, J. C., "Progress with numerical and graphical methods for practical Bayesian statistics", The Statistician, 36, pp 75-82, 1987. 


\begin{tabular}{|ll|c|c|c|c|c|c|c|c|c|}
\hline \multicolumn{2}{|l|}{$\begin{array}{l}\text { Data set } \\
\text { (no. predictions) }\end{array}$} & & & & & & & & & \\
& $\mathrm{u}$ & $.2049 \mathrm{E}$ & $.1871 \mathrm{E}$ & $.1773 \mathrm{E}$ & $.0982 \mathrm{~A}$ & $.1567 \mathrm{D}$ & $.1123 \mathrm{~A}$ & $.0982 \mathrm{~A}$ & $.1504 \mathrm{D}$ & $.1457 \mathrm{D}$ \\
$\mathrm{S} 1$ & $\mathrm{u}^{*}$ & $.1188 \mathrm{~B}$ & $.1226 \mathrm{~B}$ & $.134 \mathrm{C}$ & $.0499 \mathrm{~A}$ & $.0752 \mathrm{~A}$ & $.0499 \mathrm{~A}$ & $.0499 \mathrm{~A}$ & $.0894 \mathrm{~A}$ & $.0901 \mathrm{~A}$ \\
$(86)$ & $\mathrm{y}$ & $.1156 \mathrm{~B}$ & $.1148 \mathrm{~B}$ & $.1190 \mathrm{~B}$ & $.0795 \mathrm{~A}$ & $.1029 \mathrm{~A}$ & $.0904 \mathrm{~A}$ & $.0793 \mathrm{~A}$ & $.1148 \mathrm{~B}$ & $.1173 \mathrm{~B}$ \\
& $\mathrm{y}^{*}$ & $.1018 \mathrm{~A}$ & $.1016 \mathrm{~A}$ & $.1076 \mathrm{~A}$ & $.0775 \mathrm{~A}$ & $.0808 \mathrm{~A}$ & $.0893 \mathrm{~A}$ & $.0768 \mathrm{~A}$ & $.0901 \mathrm{~A}$ & $.0916 \mathrm{~A}$ \\
\hline & $\mathrm{u}$ & $.2717 \mathrm{E}$ & $.2713 \mathrm{E}$ & $.2705 \mathrm{E}$ & $.2645 \mathrm{E}$ & $.2596 \mathrm{E}$ & $.2717 \mathrm{E}$ & $.2704 \mathrm{E}$ & $.2382 \mathrm{E}$ & $.2372 \mathrm{E}$ \\
$\mathrm{SS3}$ & $\mathrm{u}^{*}$ & $.0982 \mathrm{C}$ & $.1042 \mathrm{D}$ & $.0978 \mathrm{C}$ & $.1057 \mathrm{D}$ & $.1122 \mathrm{D}$ & $.0987 \mathrm{C}$ & $.0997 \mathrm{C}$ & $.0864 \mathrm{~B}$ & $.1043 \mathrm{D}$ \\
$(173)$ & $\mathrm{y}$ & $.1273 \mathrm{E}$ & $.1379 \mathrm{E}$ & $.1263 \mathrm{E}$ & $.1435 \mathrm{E}$ & $.1835 \mathrm{E}$ & $.1291 \mathrm{E}$ & $.1300 \mathrm{E}$ & $.0346 \mathrm{~A}$ & $.0500 \mathrm{~A}$ \\
& $\mathrm{y}^{*}$ & $.0577 \mathrm{~A}$ & $.0664 \mathrm{~A}$ & $.0579 \mathrm{~A}$ & $.0631 \mathrm{~A}$ & $.0968 \mathrm{C}$ & $.0561 \mathrm{~A}$ & $.0558 \mathrm{~A}$ & $.0415 \mathrm{~A}$ & $.0596 \mathrm{~A}$ \\
\hline
\end{tabular}

Table 1 Kolmogorov distances for u- and y-plots for raw model and for joinedup recalibrated predictions. The letters indicate significance levels: $\mathrm{E}$ is significant at the $1 \%$ level, D at $5 \%, \mathrm{C}$ at $10 \%, \mathrm{~B}$ at $20 \%, \mathrm{~A}$ is not significant at $20 \%$. Roughly: $\mathrm{A}$ and $\mathrm{B}$ are very good, $\mathrm{C}$ is acceptable, $\mathrm{D}$ and $\mathrm{E}$ are poor.

\begin{tabular}{|c|c|c|c|c|c|c|c|c|c|c|}
\hline \multicolumn{2}{|c|}{$\begin{array}{l}\text { Data set } \\
\text { (no. predictions) }\end{array}$} & $\mathrm{JM}$ & BJM & GO & MO & DU & L & LNHPP & LV & $\mathrm{KL}$ \\
\hline \multirow{4}{*}{$\begin{array}{l}\text { S1 } \\
(86)\end{array}$} & $\mathrm{u}$ & $.2049 \mathrm{E}$ & $.1871 \mathrm{E}$ & $.1773 \mathrm{E}$ & $.0982 \mathrm{~A}$ & $.1567 \mathrm{D}$ & $.1123 \mathrm{~A}$ & $.0982 \mathrm{~A}$ & $.1504 \mathrm{D}$ & $.1457 \mathrm{D}$ \\
\hline & $\mathrm{u}^{* *}$ & $.1168 \mathrm{~B}$ & $.1197 \mathrm{~B}$ & $.1277 \mathrm{~B}$ & $.0511 \mathrm{~A}$ & $.0794 \mathrm{~A}$ & $.0507 \mathrm{~A}$ & $.0526 \mathrm{~A}$ & $.1027 \mathrm{~A}$ & $.1053 \mathrm{~A}$ \\
\hline & $y$ & $.1156 \mathrm{~B}$ & $.1148 \mathrm{~B}$ & $.1190 \mathrm{~B}$ & $.0795 \mathrm{~A}$ & $.1029 \mathrm{~A}$ & $.0904 \mathrm{~A}$ & $.0793 \mathrm{~A}$ & $.1148 \mathrm{~B}$ & $.1173 B$ \\
\hline & $y^{* *}$ & $.1109 \mathrm{~A}$ & $.1126 \mathrm{~A}$ & $.1102 \mathrm{~A}$ & $.0852 \mathrm{~A}$ & $.0762 \mathrm{~A}$ & $.0715 \mathrm{~A}$ & $.0853 \mathrm{~A}$ & $.0878 \mathrm{~A}$ & $.0916 \mathrm{~A}$ \\
\hline \multirow{4}{*}{$\begin{array}{l}\text { SS3 } \\
(173)\end{array}$} & $\mathbf{u}$ & $.2717 \mathrm{E}$ & $.2713 \mathrm{E}$ & $.2705 \mathrm{E}$ & $.2645 \mathrm{E}$ & $.2596 \mathrm{E}$ & $.2717 \mathrm{E}$ & $.2704 \mathrm{E}$ & $.2382 \mathrm{E}$ & $.2372 \mathrm{E}$ \\
\hline & $\mathrm{u}^{* *}$ & $.0820 \mathrm{~B}$ & $.0822 \mathrm{~B}$ & $.0782 \mathrm{~A}$ & $.0901 \mathrm{~B}$ & $.0916 \mathrm{~B}$ & $.0859 \mathrm{~B}$ & $.0846 \mathrm{~B}$ & $.0834 \mathrm{~B}$ & $.1006 \mathrm{C}$ \\
\hline & $y$ & $.1273 E$ & $.1379 \mathrm{E}$ & $.1263 E$ & $.1435 \mathrm{E}$ & $.1835 \mathrm{E}$ & $.1291 \mathrm{E}$ & $.1300 \mathrm{E}$ & $.0346 \mathrm{~A}$ & $.0500 \mathrm{~A}$ \\
\hline & $y^{* *}$ & $.0573 \mathrm{~A}$ & $.0693 \mathrm{~A}$ & $.0560 \mathrm{~A}$ & $.0632 \mathrm{~A}$ & $.1016 \mathrm{C}$ & $.0571 \mathrm{~A}$ & $.0557 \mathrm{~A}$ & $.0352 \mathrm{~A}$ & $.0452 \mathrm{~A}$ \\
\hline
\end{tabular}

Table 2 As Table 1 but for spline-smoothed recalibrated predictions. 
Figure 1 u-plots for $\mathrm{JM}$ and $\mathrm{LV}$ model predictions of $\mathrm{T}_{51}$ through $\mathrm{T}_{136}$, Musa $\mathrm{S} 1$ data [17].

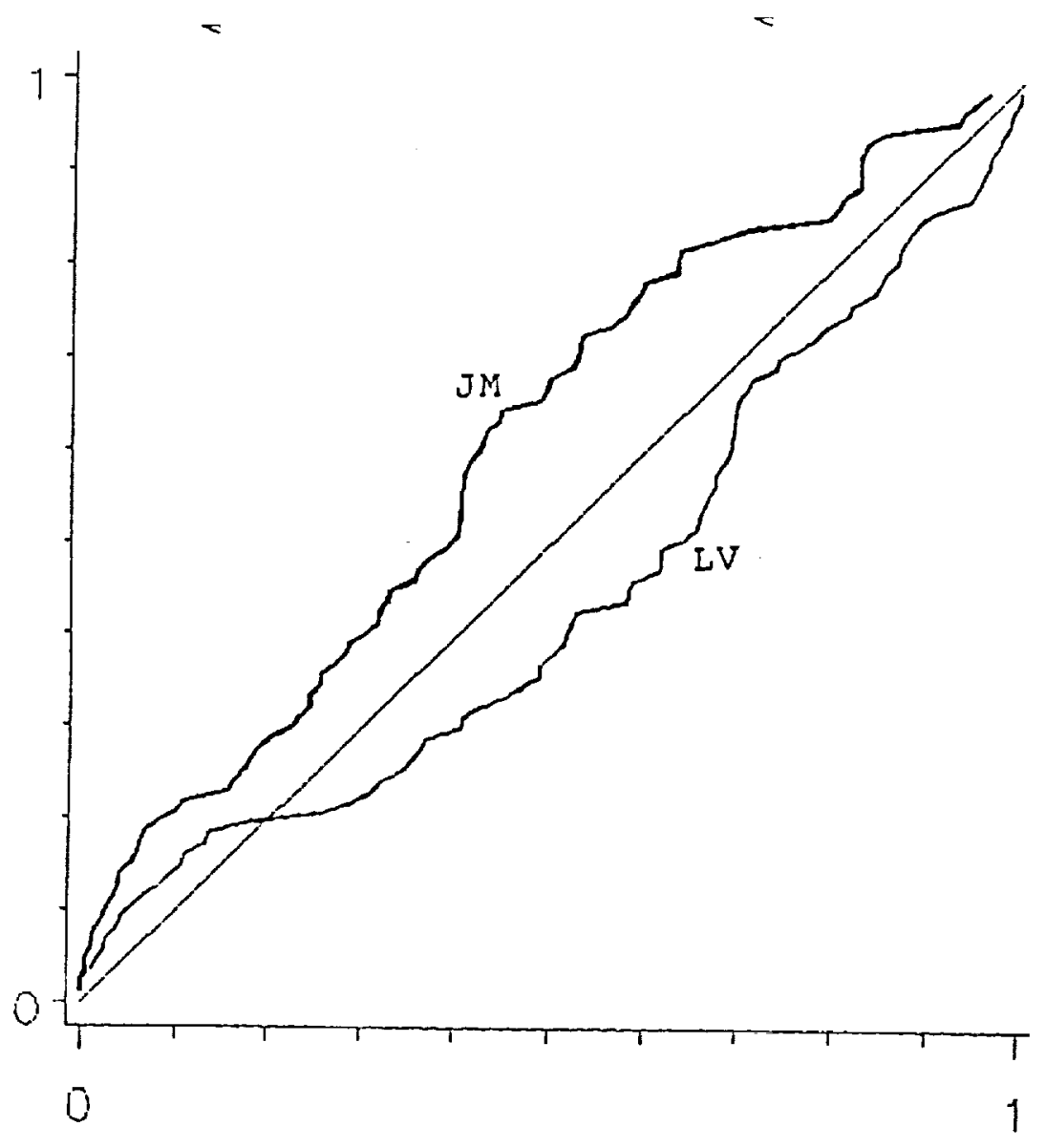


Figure 2 Method of drawing the joined-up step recalibrating function, $G_{i}{ }^{*}$. Here there are $\mathrm{r} u$-points and each step is of size $1 /(\mathrm{r}+1)$.

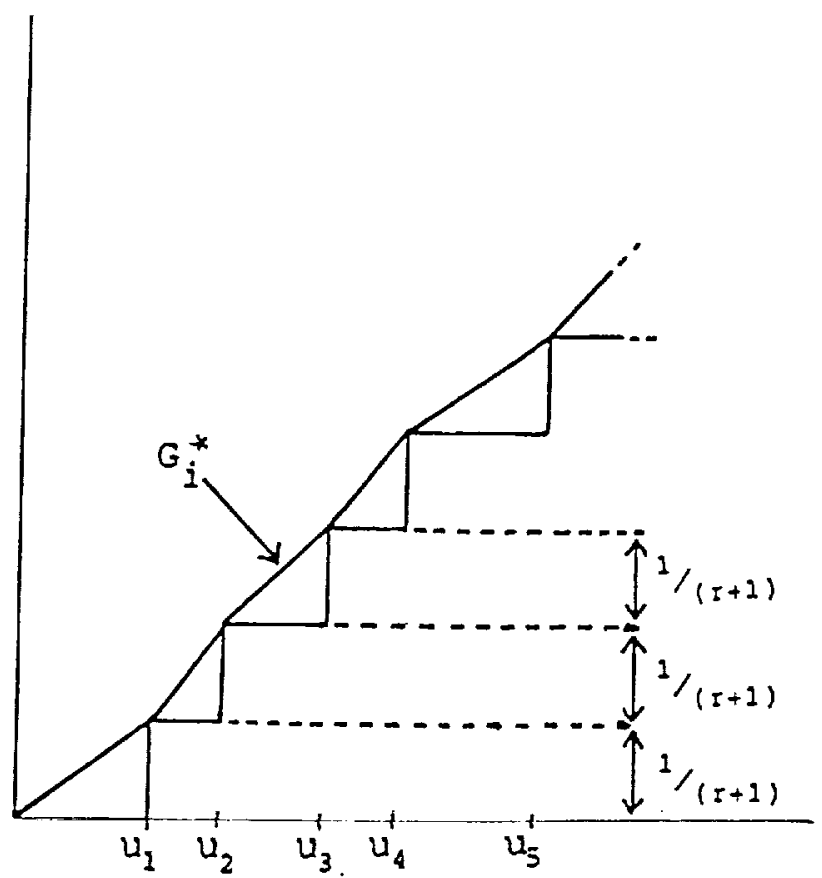


Figure 3 Predictive medians of $T_{51}$ through $T_{136}$, raw and recalibrated using joined-up recalibrator, $\mathrm{G}_{i}^{*}$, for Musa System 1 data [17].

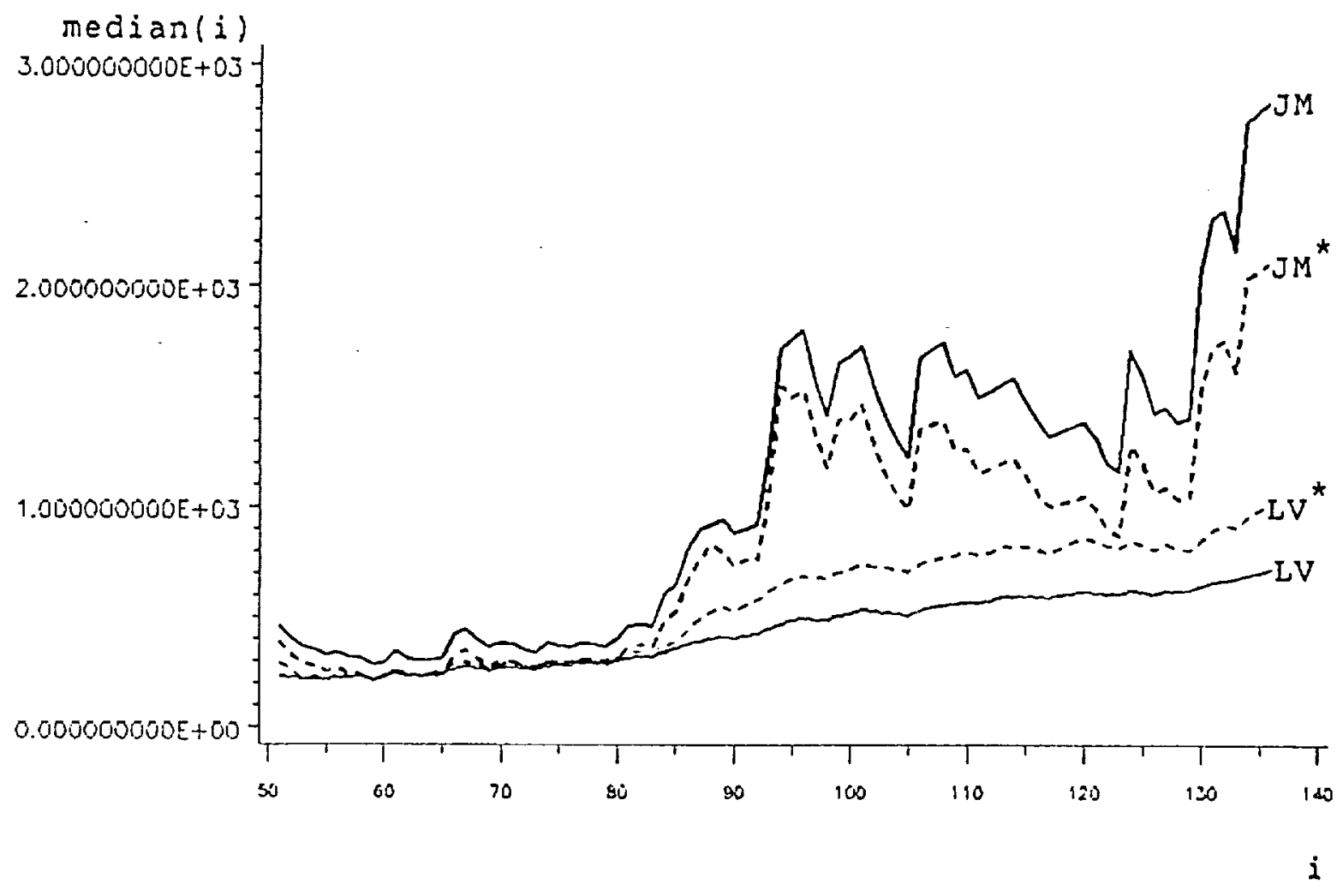


Figure 4 Raw predictive medians of $T_{106}$ through $T_{278}$, for all nine models, Musa SS3 data [17].

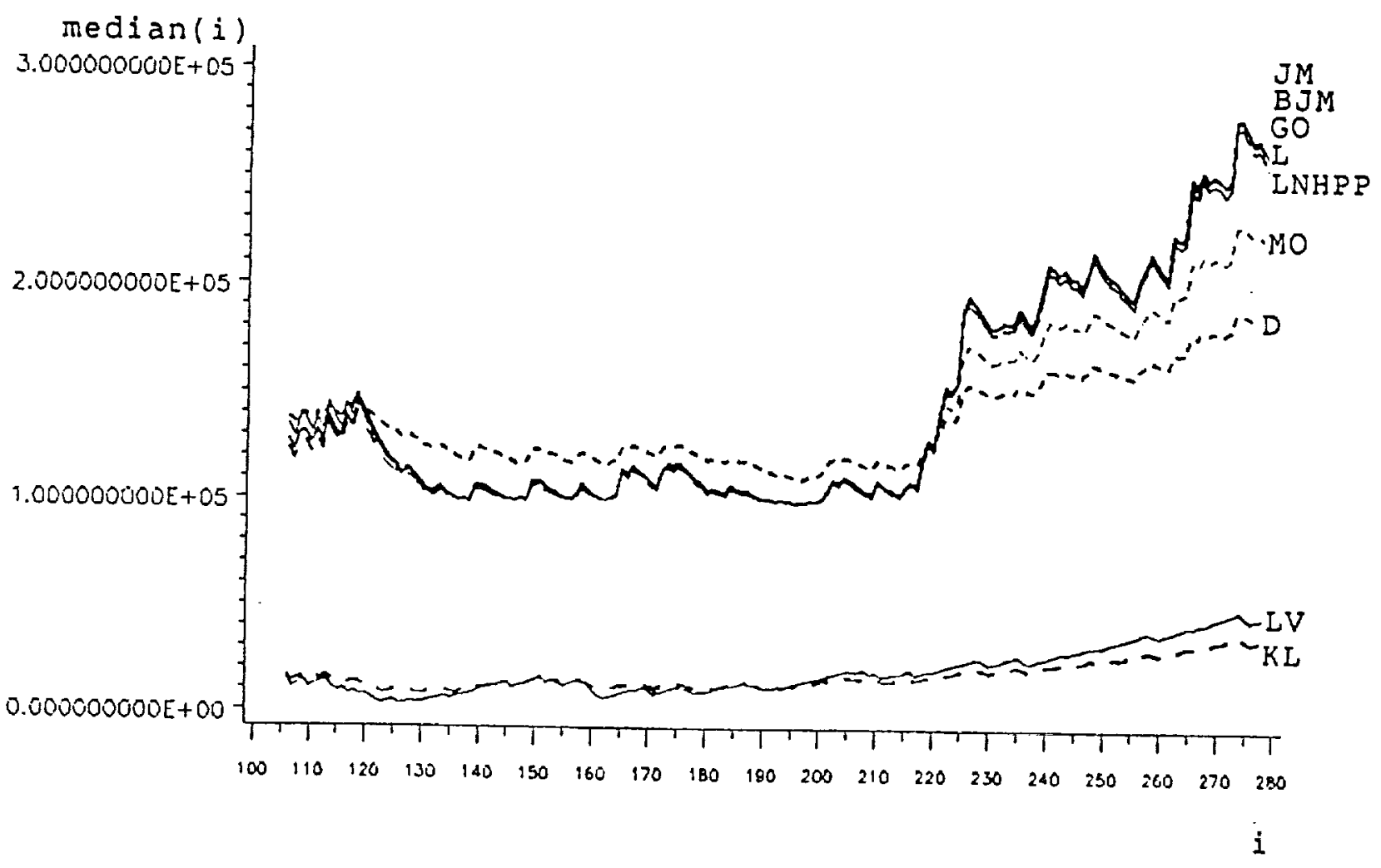


Figure 5 u-plots for raw predictions of $T_{106}$ through $T_{278}$, JM and LV models, Musa SS3 data [17].

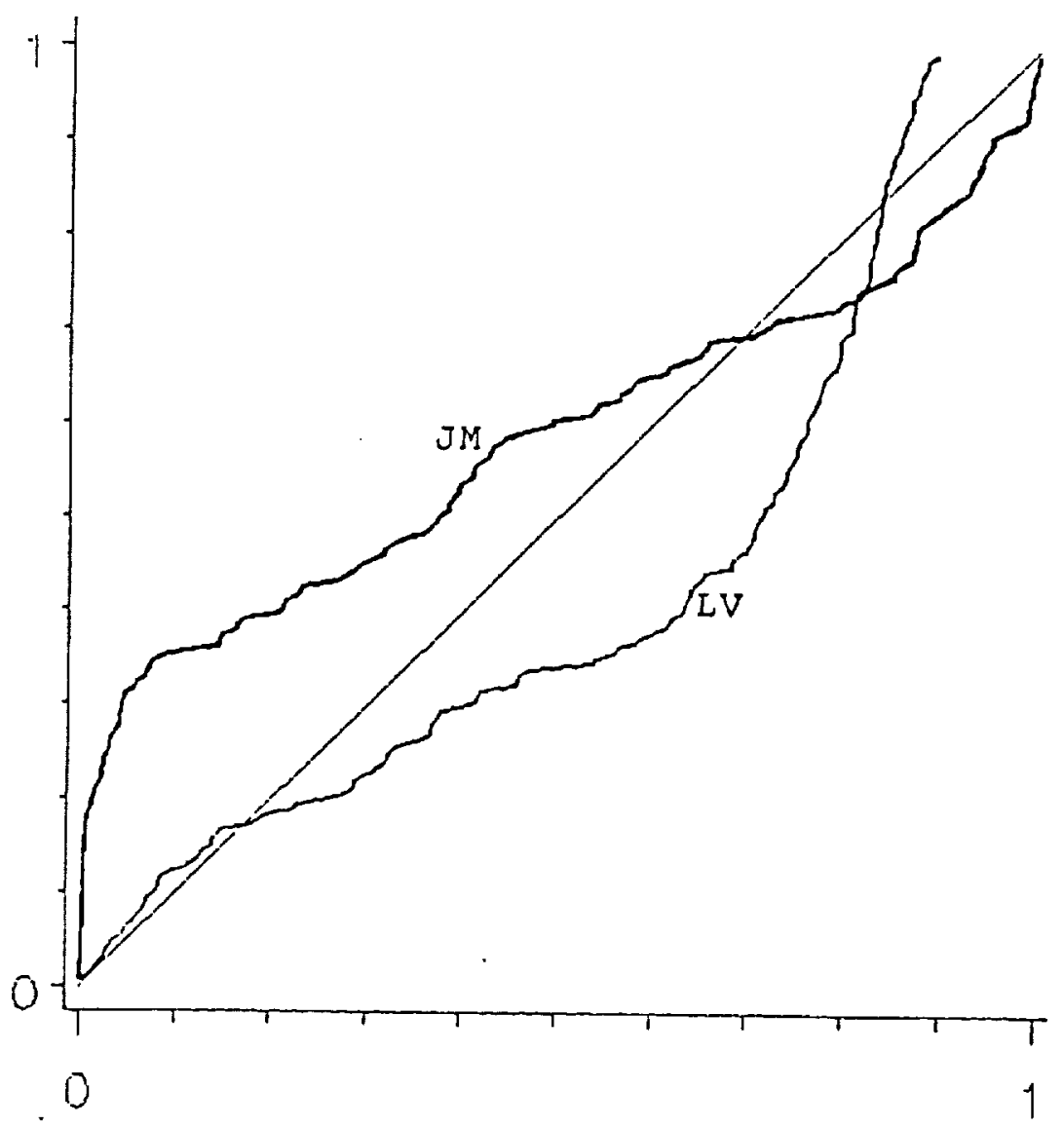


Figure $6 \mathrm{u}^{*}$-plots for (joined-up) recalibrated predictions of $T_{106}$ through $T_{278}$, JM and LV models, Musa SS3 data [17].

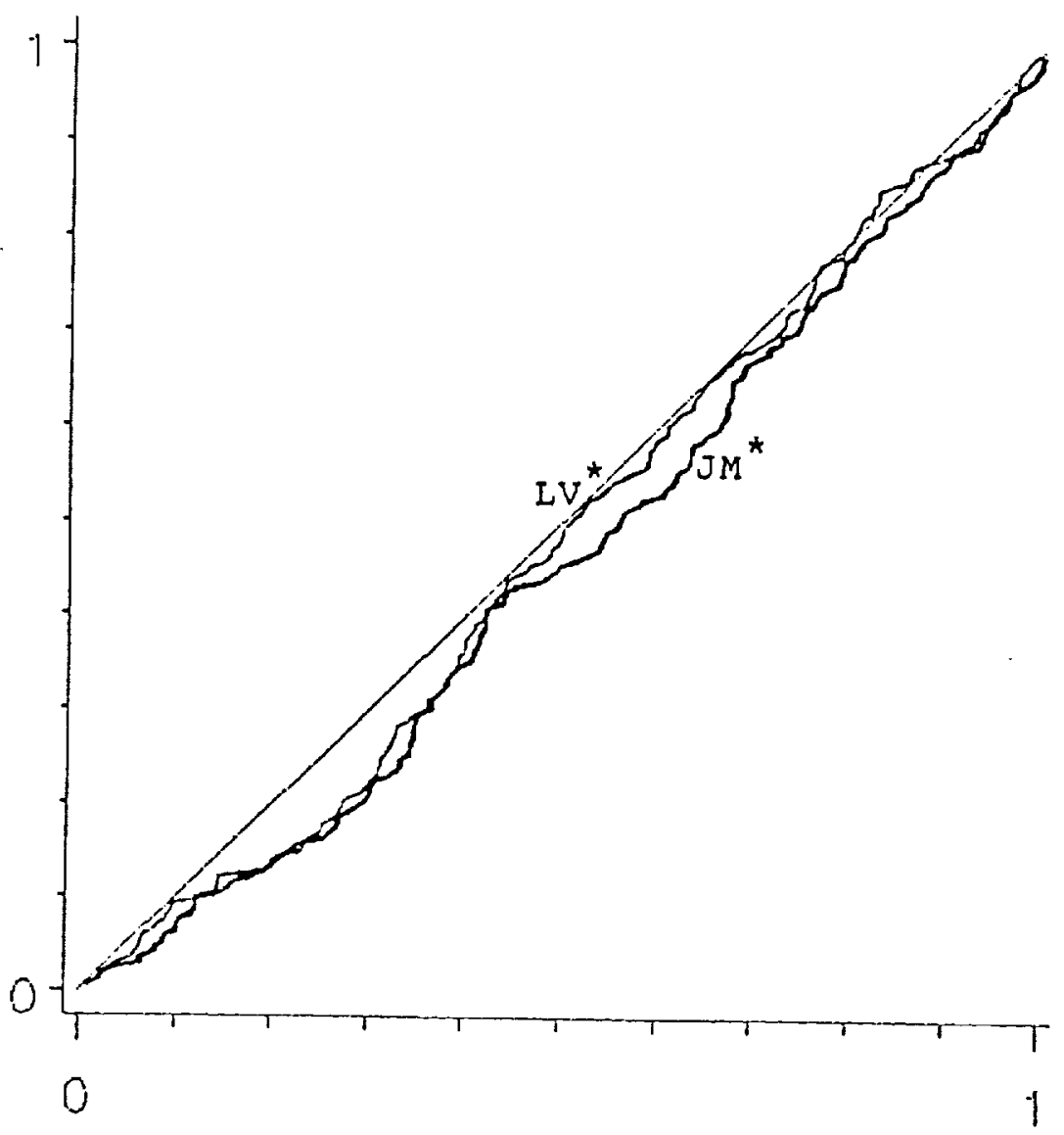


Figure 7 Medians of (joined-up) recalibrated predictions of $T_{106}$ through $T_{278}$, $\mathrm{JM}$ and LV models, Musa SS3 data [17].

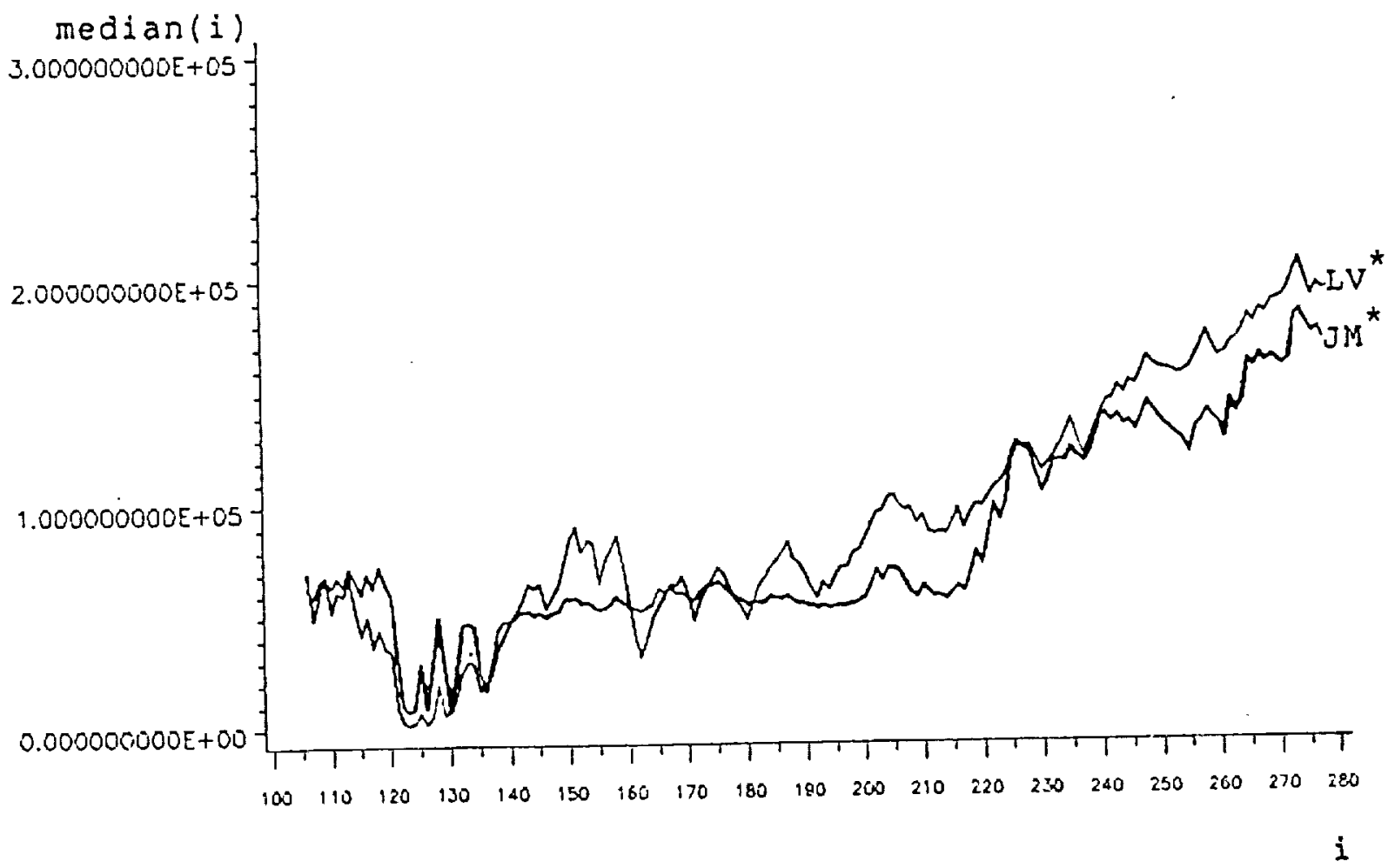


Figure 8a The joined-up recalibrator, $\mathrm{G}_{278}{ }^{*}$, based on a u-plot of 188 points, for the LV modeI on Musa SS3 data

$<$

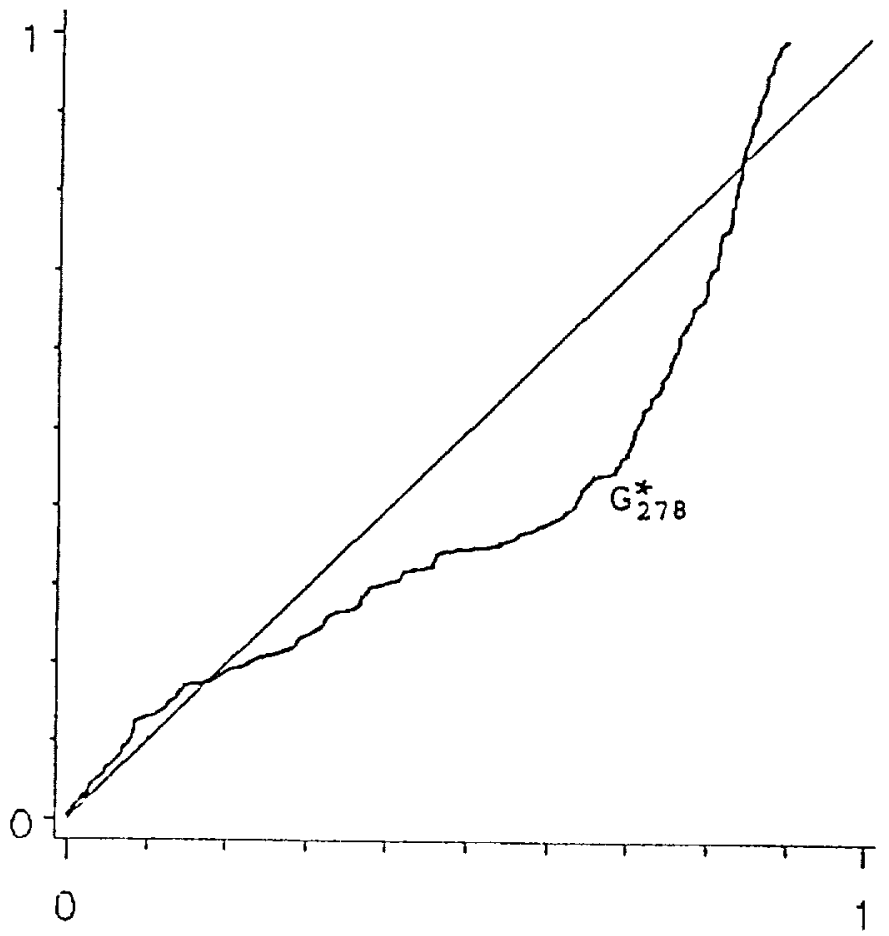


Figure 8b The derivative of $G_{278}{ }^{*}$ in Figure 8a.

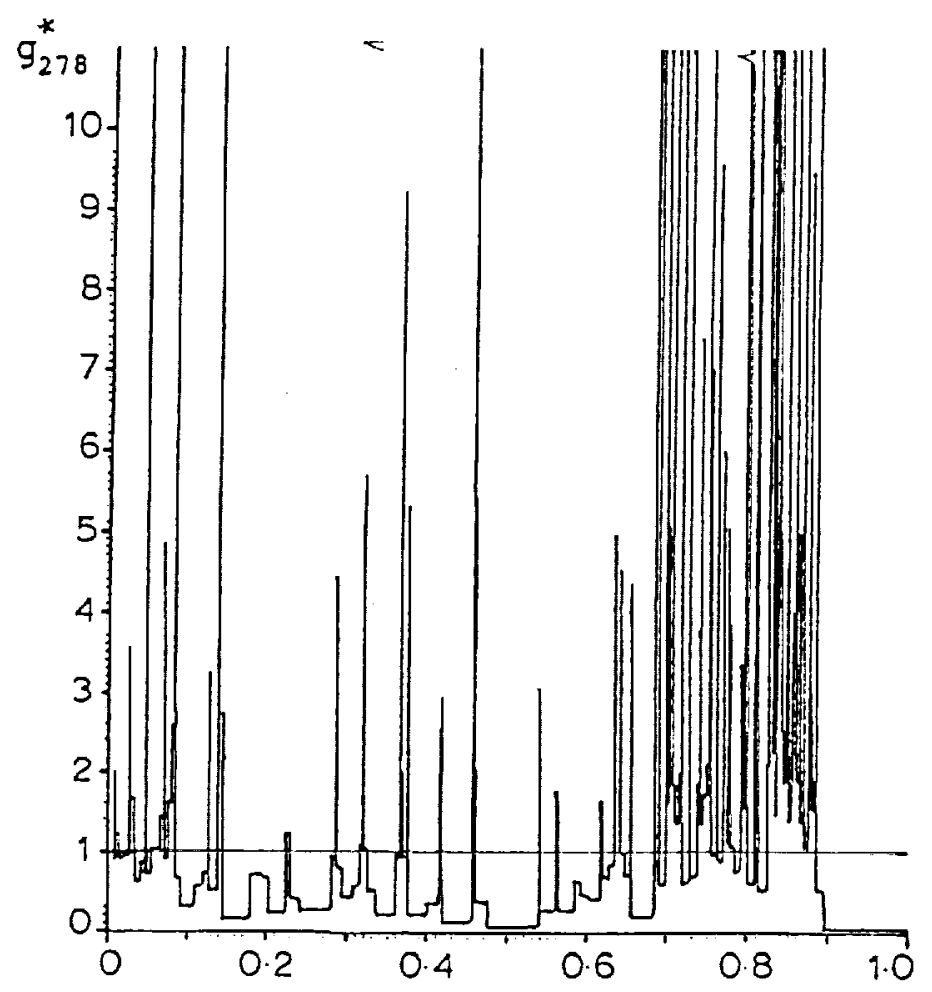


Figure 9 Medians of spline recalibrated predictions of $\mathrm{T}_{106}$ through $\mathrm{T}_{278}$, JM and LV models, Musa SS3 data. Note closeness to results in Figure 7 for joined-up recalibration.

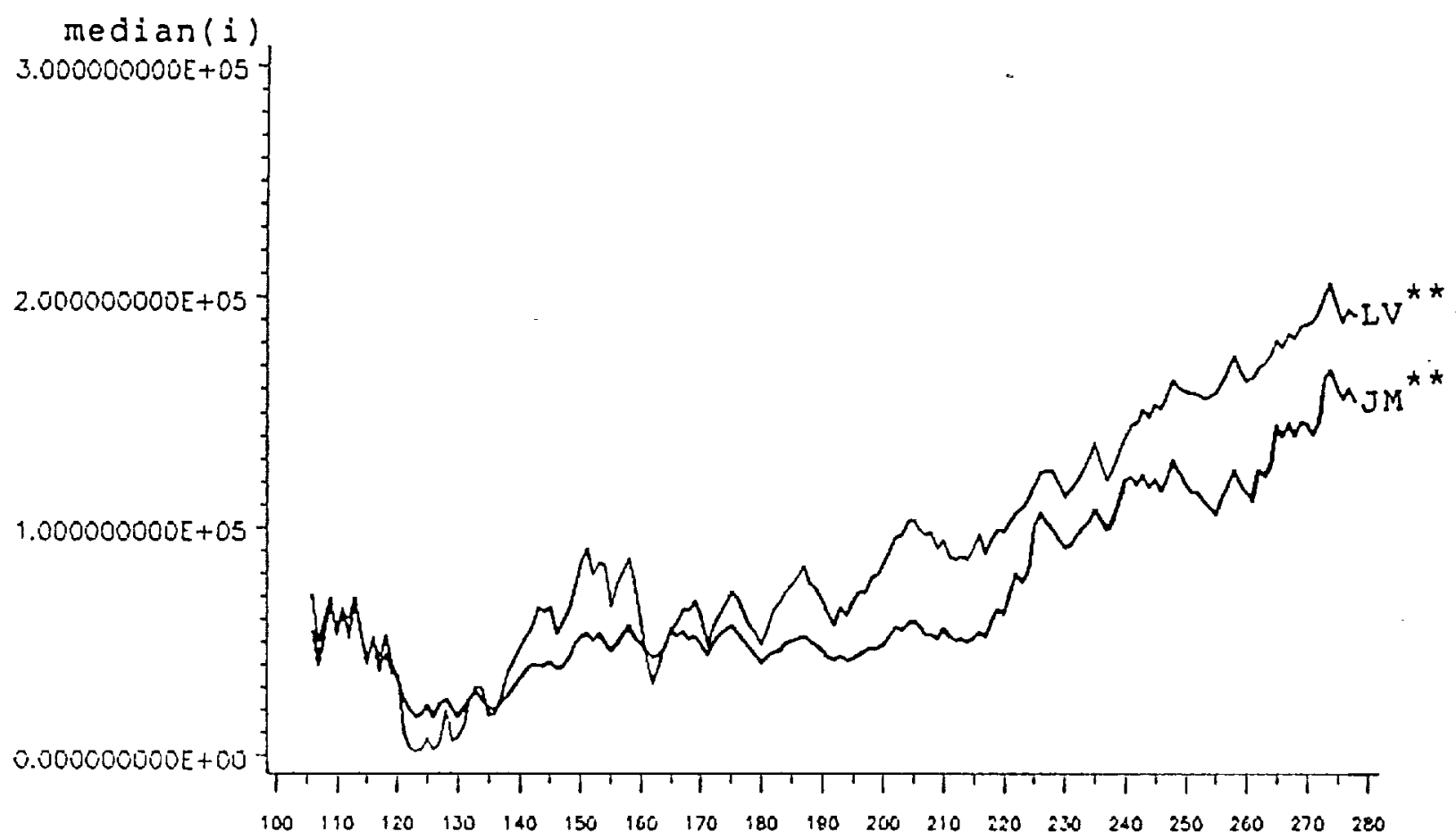


Figure 10 Plot of logPLR against $i$, showing comparison of predictive accuracy between the each type of recalibration and the raw predictions; $\mathrm{N}$ and LV models, Musa SS3 data.

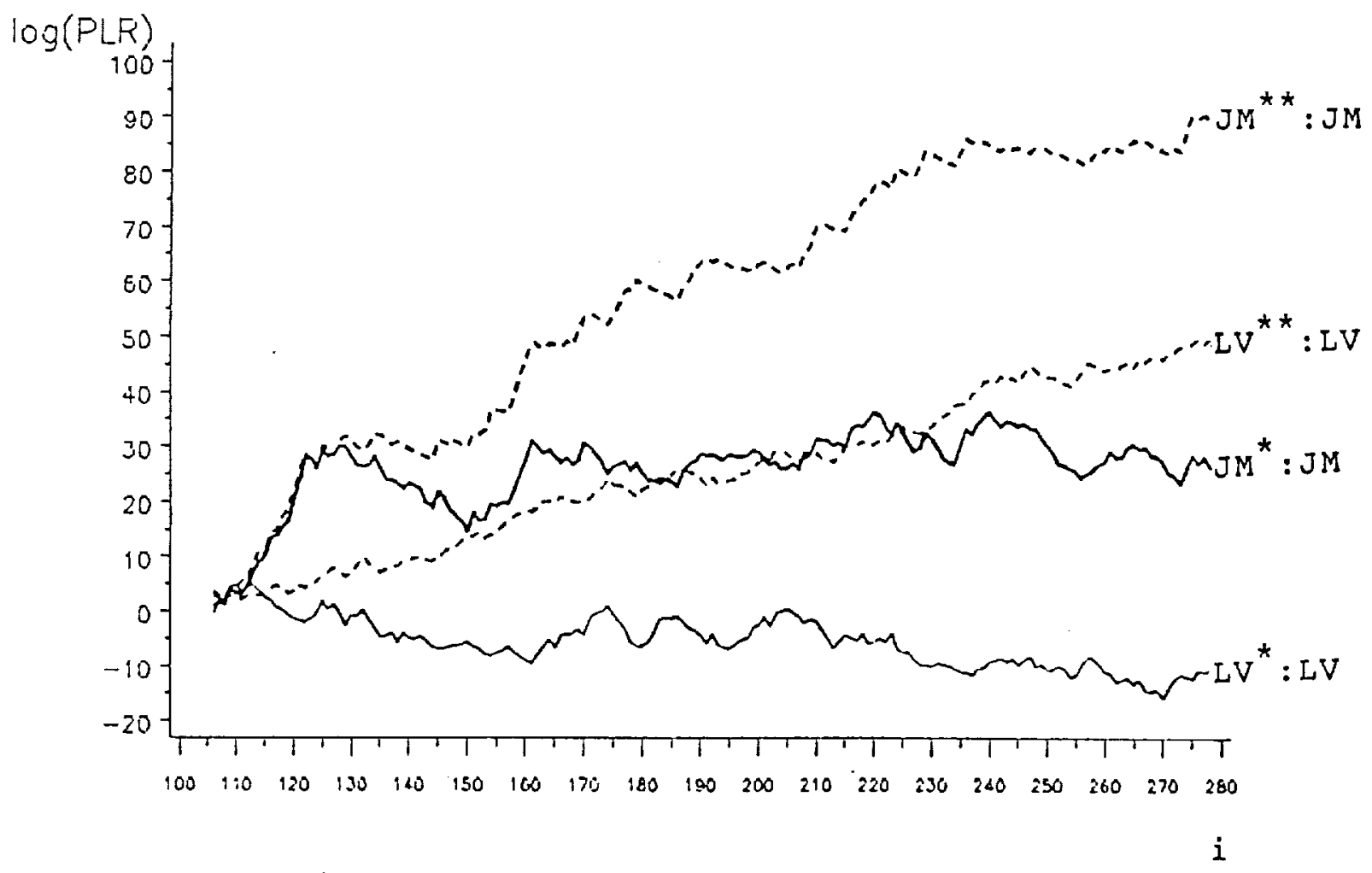


Figure 11 Examples of individual spline recalibrated predictive probability density functions for $T_{278}$, using $\mathrm{N}$ and LV models on Musa SS3 data. Note great difference between raw predictions, and closeness of recalibrated.

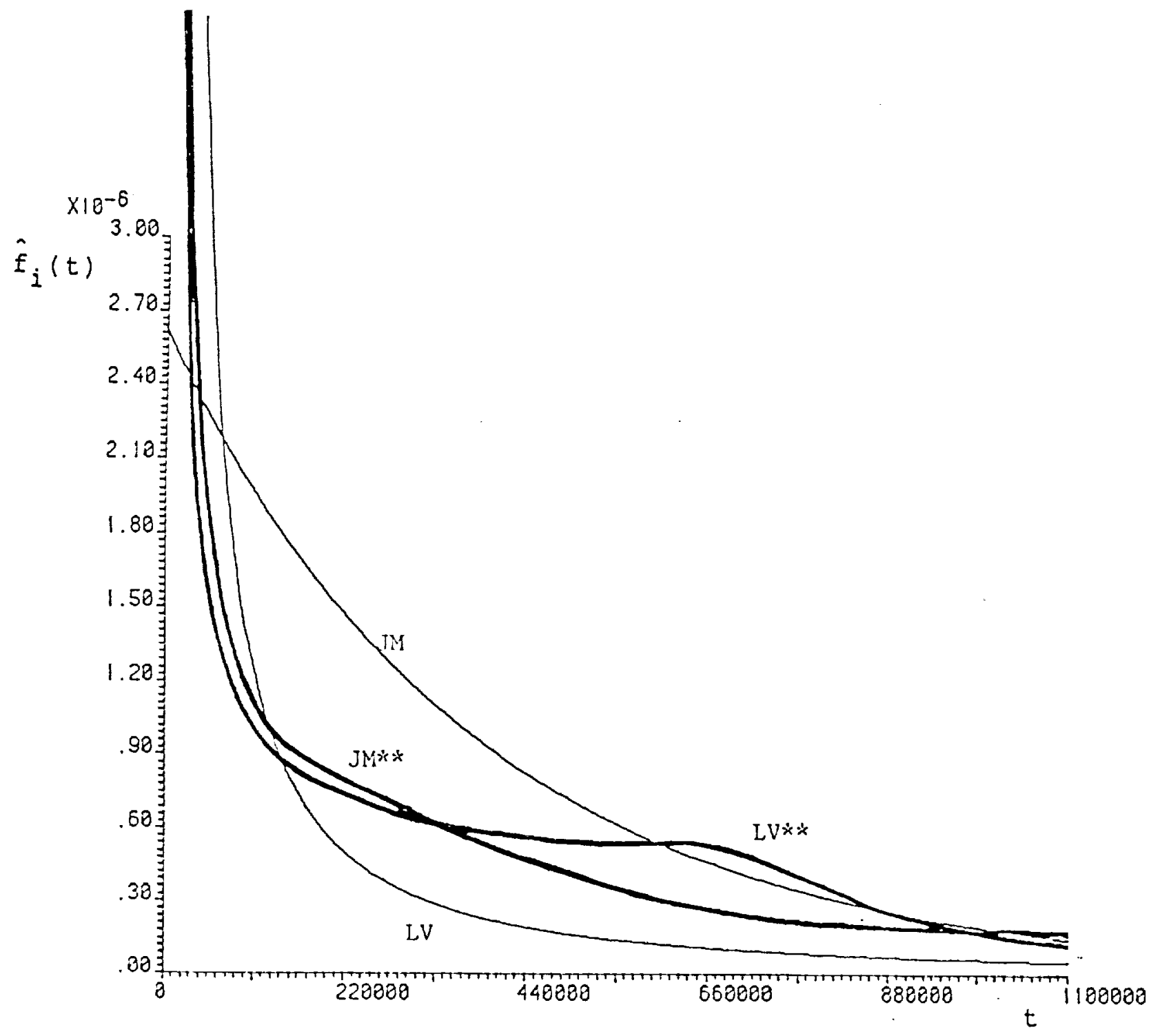


Figure 12 As Figure 11, but for $T_{121}$, Musa $S 1$ data. Note, again, closeness of the two recalibrated predictions and how these differ greatly from the (very different) raw predictions.

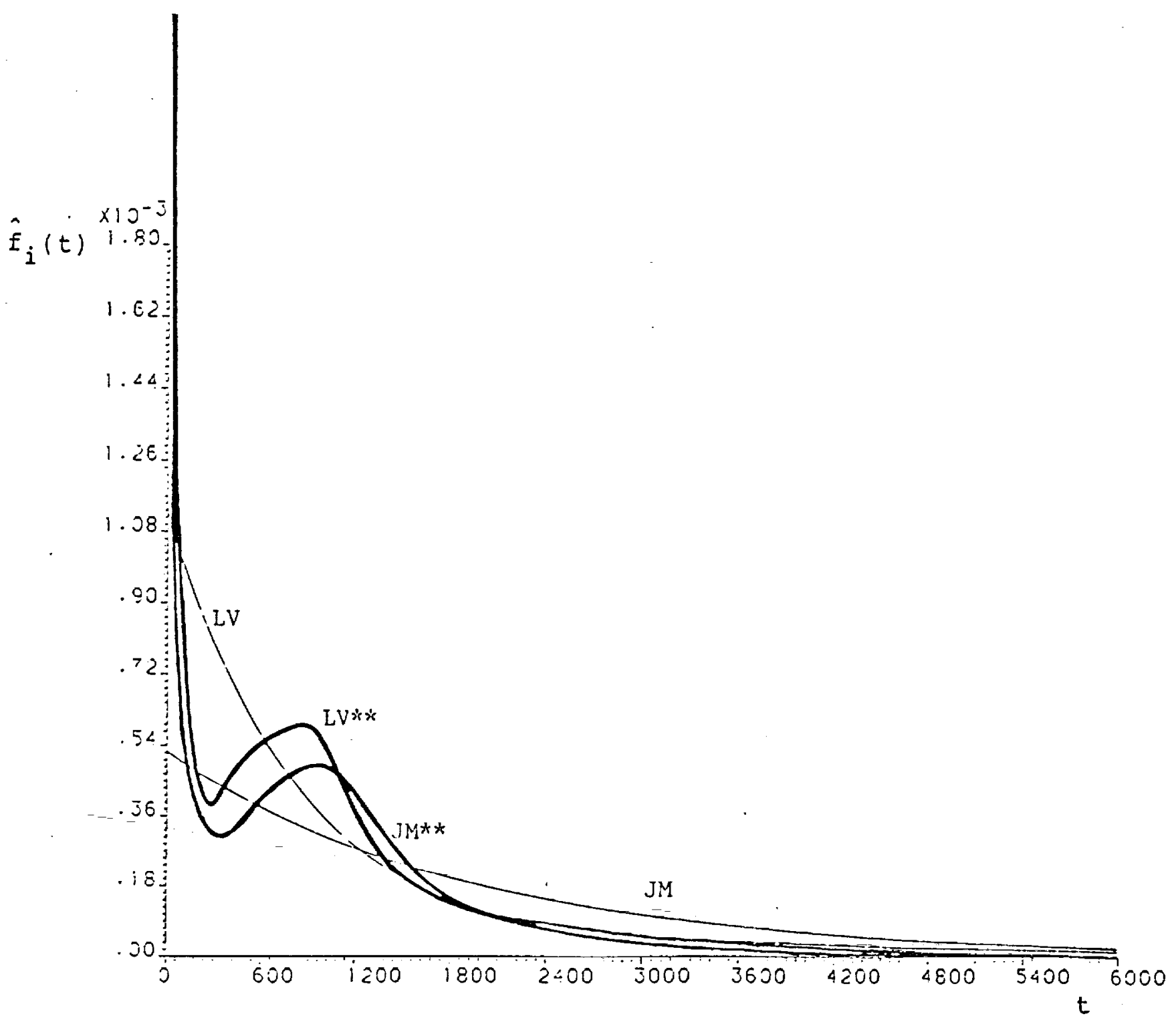

ORIGINAL PAGE IS OF POOR QUALITY 